Journal of

Molecular Microbiology

and Biotechnology

\title{
In vivo Activation of Tetracycline Rep ressor by Cre/lox-Mediated Gene Assembly
}

\author{
Ralph Bertram ${ }^{a}$ b Martina Kolb ${ }^{a}$ Wolfgang Hillen ${ }^{b}$ \\ a Lehrbereich mikrobielle Genetik, Eberhard-Karls-Universität Tübingen, Tübingen, und ${ }^{\text {b } L e h r s t u h l ~ f u ̈ r ~}$ \\ Mikrobiologie, Friedrich-Alexander-Universität Erlangen-Nürnberg, Erlangen, Deutschland
}

\section{Key Words}

Tetracycline repressor - Gene regulation - Site-specific recombination - Bacillus subtilis

\begin{abstract}
Tetracycline repressor (TetR) bears an unstructured loop region between helices $\alpha 8$ and $\alpha 9$, which is moderately permissive to amino acid exchanges and length variations. Recognition sites for the site-specific recombinases Flp (FRT) or Cre (lox) were inserted in-frame into tetR, substituting some of this loop's codons. A number of the deduced TetR variants displayed efficient regulation in vivo, thus allowing the establishment of a new mode of TetR activation on the genetic level. Chromosomally encoded tetR in Bacillus subtilis was disrupted and inactivated by insertion of a lox66-aphAllllox71 kanamycin resistance cassette. Marker excision by Cre recombinase led to the assembly of a novel tet $R$ allele. The encoded regulator, termed Tet $\mathrm{R}^{\mathrm{lox} 72 / 1}$, is distinguished from wt-TetR by a slightly elongated and altered $\alpha 8-\alpha 9$ loop only, harboring an amino acid stretch encoded by lox72. Despite decreased intracellular protein amounts, TetR $\mathrm{R}^{\mathrm{lox} 72 / 1}$ displayed efficient in vivo activity in B. subtilis and $E$. coli, indistinguishable from that of wt-TetR. These results underline the sequence flexibility of TetR in the $\alpha 8-\alpha 9$ loop and demonstrate the possible use of the regulator as a read-out tool for the activity of site-specific recombinases.
\end{abstract}

Copyright $\odot 2009$ S. Karger AG, Basel

\section{Introduction}

Tetracycline-dependent gene regulation (tet regulation) is a frequently applied means of gaining transcriptional control over genes in prokaryotes and eukaryotes [reviewed in Berens and Hillen, 2003 and in Bertram and Hillen, 2008]. The origin of tet regulation can be traced back to the Tc resistance determinants tet(B) of Tn 10 [Hillen and Schollmeier, 1983] and tet(D) of plasmid RA1 [Unger et al., 1984]. Both comprise tetR-tetA divergons, in which the tc antiporter TetA is transcriptionally controlled by tetracycline repressor (TetR) [reviewed in Hillen and Berens, 1994]. Binding of TetR to its cognate DNA sequence $t e t O$ can be reversibly suspended by administration of subinhibitory doses of the antibiotic tetracycline (Tc). This results in tet $A$ expression with $\mathrm{Tc}$ or tet $A$ repression without Tc. The genetic background of this system in $\mathrm{Tn} 10$ is well understood and involves three partly intertwined promoters and two tet $O$ sites in the intergenic tetR-tetA region [reviewed in Grkovic et al., 2002 and in Hillen and Berens, 1994]. Negative autoregulation of tet $R$ results in a balanced amount of the regulator. This

Abbreviations: $\mathrm{Ap}=$ Ampicillin; Atc $=$ anhydrotetracycline; $\mathrm{Cm}=$ chloramphenicol; Em = erythromycin; FRT = Flp recognition target; $\mathrm{Km}=$ kanamycin; lox = locus of cross-over of P1; SSR = site-specific recombination; $\mathrm{Tc}=$ tetracycline.

\section{KARGER}

Fax +41613061234

E-Mail karger@karger.ch

www.karger.com (c) 2009 S. Karger AG, Basel

$1464-1801 / 09 / 0173-0136 \$ 26.00 / 0$

Accessible online at:

www.karger.com $/ \mathrm{mmb}$
Ralph Bertram

Lehrbereich mikrobielle Genetik

Eberhard Karls Universität Tübingen, Waldhäuser Strasse 70/8

DE-72076 Tübingen (Germany)

Tel. +497071297 8855, Fax +497071295 937, E-Mail ralph.bertram@uni-tuebingen.de 
ensures both sensitive induction at low Tc levels and tight regulation in the absence of the drug. For applied tet regulation in bacteria, the conventional set-up consists of a promoter containing one or two tet $O$ sequences and a downstream target gene, the activity of which can be modulated by Tc and TetR, encoded in cis or trans. Some of the most efficient settings for applied tet regulation in bacteria rely on constitutively high tet $R$ expression [Lutz and Bujard, 1997; Skerra, 1994] for two major reasons. First, the tightness of repression correlates to the abundance of TetR. Second, the use of the less antibiotic Tc derivative anhydro-Tc (ATc), which binds TetR with at least 35-fold higher affinity than Tc [Degenkolb et al., 1991], allows for complete induction even at high intracellular TetR amounts. To enable efficient transcription of a tet-regulated gene, available TetR is induced by (A)Tc, which is added to the growth medium. Alternatively, target gene expression can also be achieved without inducer, given that no TetR or only marginal amounts of it are present in the cell. To this end, tetR can e.g. itself be transcriptionally repressed by a second inducible system [Bertram et al., 2005; Kamionka et al., 2005], such that repression of the target gene requires expression of tet $R$. In the present study, we describe a novel kind of tet $R$ activation in vivo that is not based upon transcriptional or translational control, but exploits a particular structural feature of the regulator. TetR's active form consists of two identical monomers which fold to form $10 \alpha$-helices connected by turns and loops [Hinrichs et al., 1994; Orth et al., 1998, 2000]. Of these, the loop sequence between helices $\alpha 8$ and $\alpha 9$ is not resolved in published TetR structures. Earlier studies have shown that this stretch in the TetR variants of tet(B) and tet(D) is permissive for alterations to a certain extent. This pertains to both length and amino acid composition [Berens et al., 1997; Kintrup et al., 2000; Scholz et al., 2001]. The second prerequisite for the described approach is the availability of a site-specific recombination (SSR) system for the organism of choice. The most commonly applied of these in mammalian and bacterial genetics are the yeast derived Flp/FRT system [reviewed in Schweizer, 2003] and Cre/lox, originating from bacteriophage P1 [reviewed in Sauer, 2002]. Both are very similar in structural and functional terms, requiring 34bp recognition sequences, which serve as binding sites and substrates for the respective recombinase. Upon Flp or Cre treatment, respectively, a region between two collinear lox or FRT sequences within a circular or linear DNA molecule is precisely excised [reviewed in Grindley et al., 2006]. A number of functional variant lox sites have been identified in the past. Among these are lox66 and lox71, each with 5-bp exchanges compared to wt-loxP. After Cre-mediated recombination between these two sites, a sequence called lox72 is obtained, which is mutated at ten positions [Albert et al., 1995]. Although lox and FRT sites are functionally noncoding, the addition of two basepairs renders them able to encode dodecapeptides. In this study, we analyzed the potential of tet $R$ alleles containing internal lox or FRT sequences for encoding functional TetR variants. Furthermore, the Cre/lox system was exploited to assemble a functional gene from an artificially split, bipartite tet $R$-like sequence within the Bacillus subtilis chromosome. To this end, a floxed (flanked by lox) resistance marker was first used to disrupt wt-tet $R$ and was then excised by Cre. This yielded an ORF harboring one in-frame lox sequence, whose deduced TetR variant, containing an altered $\alpha 8-\alpha 9$ loop sequence, was functional in vivo with full efficiency.

\section{Results}

\section{In vivo Activity of TetR Variants Harboring SSR} Recognition Sites

The loop region between helices $\alpha 8$ and $\alpha 9$ (fig. 1a) consists of 15 residues (positions 152 to 167) in TetR and is identical to that of TetR(D). Previous studies have shown that length and sequence variations of this region are tolerated to a certain extent. The first aim was to replace codons 161 to 167 for different SSR recognition sites, thereby maintaining translational read-through. In vivo activity analyses of these new TetR variants should shed further light on the permissivity of this region. FRT- or lox72-containing sequences were cloned into the tet $R$ expression vector pWH1926, yielding pWH1926-F1, pWH1926-L1 and pWH1926-L2 as the desired constructs as well as pWH1926-F1-inv, pWH1926-L1-inv and pWH1926-L2inv, in which the fragments inserted in opposite orientation. Due to the position of the two PstI sites used for cloning within tetR, the deduced proteins of the 'inv' constructs bore sequence alterations not only affecting the loop, but also the helix $\alpha 9$ region. In the case of L2-inv, this causes premature termination by an ochre stop codon. Hence, this construct, termed tetR $R^{l o x 72 / 2-i n v}$, encodes a truncated TetR-like polypeptide of 170 amino acids only. All other ORFs code for full-length TetR monomers of 214 or 215 residues, which are designated as follows: Tet $R^{\text {FRT }}$ (FRT site), TetR $\mathrm{R}^{\operatorname{lox} 72 / 1}$ (lox72, first reading frame), Tet$\mathrm{R}^{\text {lox72/2 }}$ (lox72, second reading frame), Tet $\mathrm{R}^{\mathrm{FRT} \text {-inv }}$ and Tet$\mathrm{R}^{\text {lox72/1-inv }}$. The altered sequence stretches of the variants, as well as their in vivo activities in E. coli, are depicted in 
Table 1. Bacterial strains or plasmids used in this study

Relevant characteristics or genotype

Reference

Strains

E. coli $\mathrm{DH} 5 \alpha$

E. coli WH207 $\lambda$ tet50

B. subtilis WH557

B. subtilis WH558

B. subtilis WH560

B. subtilis RAB 100

B. subtilis RAB 101

B. subtilis RAB102

\section{Plasmids}

pCrePA

pWH1925

pWH1926

pWH1926-F

pWH1926-F-inv

pWH1926-L1

pWH1926-L1-inv

pWH1926-L2

pWH1926-L2-inv

pWH1926-TLKLT

pWH1411BD

pWH1411-TLKLT

pWH1411-TLKLTextd
recA1 endA1 gyrA96 thi relA1 hsdR17 $\left(\mathrm{r}_{\mathrm{K}}{ }^{-}, \mathrm{m}_{\mathrm{K}}{ }^{+}\right)$supE44 Ф80dlacZs $\Delta$ lacU169

galK rpsL thi $\Delta$ lacX74 recA13 Tn10 tetA-lacZ transcriptional fusion bet $^{+} \mathrm{gam}^{+} \mathrm{cIII} \mathrm{I}^{+} \mathrm{II}^{+}$

trpC2 lacA::Pt17-tetR lox66-aphAIII-lox71

trpC2 lacA::Pt17-tetR lox66-aphAIII-lox71 amyE:: $\mathrm{P}_{\mathrm{xyl} / \mathrm{tet}}$-lacZ

$\operatorname{trp} C 2$ amyE:: $\mathrm{P}_{\mathrm{xyl} / \mathrm{tet}}-\mathrm{lac} Z$

trpC2 lacA::Pt17-tetR-lox72 amyE::P $\mathrm{P}_{\mathrm{xyl} / \mathrm{tet}}$-lacZ

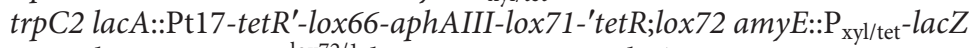

trpC2 lacA::Pt17-tet $R^{\operatorname{lox} 72 / 1}$ lox72 amyE:: $\mathrm{P}_{\mathrm{xy} 1 / \mathrm{tet}}$-lacZ

$\mathrm{Em}^{\mathrm{R}}, \mathrm{P}_{\text {pagA }}$-cre thermosensitive, expression of cre in Bacillus

$\mathrm{Ap}^{\mathrm{R}}$, pUC19 derivative, no tet $R$

$A^{R}$, pUC19 derivative, constitutive expression of tet $R$

pWH1926 derivative, tet $^{\mathrm{FRT}}$

pWH1926 derivative, tet $^{\text {FRT-inv }}$

pWH1926 derivative, tet $^{\text {lox } 72 / 1}$

pWH1926 derivative, tet $R^{\text {lox72/1-inv }}$

pWH1926 derivative, tet $^{\text {lox72/2 }}$

pWH1926 derivative, tet $R^{\text {lox72/2-inv }}$

pWH1926 derivative, tetR'-lox66-aphAIII-lox71-'tetR

$\mathrm{Cm}^{\mathrm{R}}$, pACYC177 derivative, tet $R$

pWH1411BD derivative, tet $R^{\prime}$-lox66-aphAIII-lox71-'tet $R$

pWH1411BD derivative, tetR'-lox66-aphAIII-lox71-'tetR 'lacA'
Hanahan, 1983

Smith and Bertrand, 1988;

Wissmann et al., 1991

Bertram et al., 2005

Bertram et al., 2005

Bertram et al., 2005

this study

this study

this study

Pomerantsev et al., 2006

Bertram et al., 2004

Kamionka et al., 2006

this study

this study

this study

this study

this study

this study

this study

Scholz et al., 2003

this study

This study

$\mathrm{R}=$ Resistant.

figure $1 \mathrm{~b}$. Intriguingly, all three TetR variants in which only the loop region was altered displayed full inducibility with ATc. Also, the repression capacities of TetR ${ }^{\text {FRT }}$ and TetR ${ }^{\text {lox72/1 }}$ were almost identical to that of wt-TetR, but were diminished in case of TetR $\mathrm{R}^{\text {lox} 72 / 2}$. This sharply contrasts with the behavior of the '-inv' constructs, none of which has regulatory properties. The truncated Tet $R^{\text {lox72/2-inv }}$ is inactive, whereas Tet $R^{\mathrm{FRT}}$ and $\mathrm{Tet}^{\text {lox72/1 }}$ apparently bind to tet $O$, but are induction deficient.

\section{A Vector for Chromosomal Integration of a Split}

tetR-Like Sequence into B. subtilis

The next aim was to provide a model system through which an artificially interrupted tet $R$ variant could be activated upon treatment with a site-specific recombinase. Based upon the results described above, a lox66-(marker gene)-lox71 cassette between two parts of a tetR gene was intended to be excised in vivo, to obtain $t e t R^{l o x 72 / 1}$ as the final product. The corresponding manipulations were ac- complished within the chromosome of B. subtilis using the Cre/lox system for a number of reasons. First, B. subtilis is conveniently accessible to transformation and integration of foreign DNA into the chromosome by double homologous recombination [Fernandez et al., 2000]. Second, strains of this species are available to monitor the in vivo activity of TetR variants [Bertram et al., 2005; Kamionka et al., 2005]. Finally, a Cre/lox setting has recently been described for use in Bacillus species [Pomerantsev et al., 2006; Yan et al., 2008]. In order to clone an integration vector, an aphAIII cassette conferring $\mathrm{Km}$ resistance was amplified and modified by PCR. Thereby, it became flanked by lox 66 and seven codons corresponding to positions 168-174 of TetR helix $\alpha 9$ upstream and by a lox71 site downstream. This product was inserted between the PstI sites of tetR, yielding a tet $R^{\prime}$-lox66-aphAIII-lox71-'tetR sequence termed tlklt. After subcloning and minor modifications (see 'Material and Methods'), the final B. subtilis integration vector pWH1411-TLKLTextd was obtained. 
Table 2. Primers used in this study (FRT and lox sites in boldface)

\begin{tabular}{|c|c|}
\hline Oligonucleotide & Sequence $\left(5^{\prime} \rightarrow 3^{\prime}\right)$ \\
\hline DP3 & GCATTATTGCGCAGAAAT \\
\hline DP8neu & CAGCGGTCAGTCATTTTA \\
\hline flp_fw & TTGAAGTTCCTATACTTTCTAGAGAATAGGAACTTCCCGCTATTGCGGGAAGCGCTGCA \\
\hline flp_rev & GCGCTTCCCGCAATAGCGGGAAGTTCCTATTCTCTAGAAAGTATAGGAACTTCAATGCA \\
\hline Km_66_inv & $\begin{array}{l}\text { GCTACTGCAGCGCTTCCCGCAATAGCGGGATACCGTTCGTATAATGTATGCTATACGAAGTTATGC- } \\
\text { GAACCATTTGAGGTGATAGGT }\end{array}$ \\
\hline Km_71_inv & GCTACTGCAGCCTACCGTTCGTATAGCATACATTATACGAAGTTATGAGTATGGACAGTTGCGGATG \\
\hline$\underline{\mathrm{Km} 1}$ & ATGACGGACAGCCGGTATAAAGG \\
\hline $\mathrm{Km} 2$ & AGATGTTGCTGTCTCCCAGGTCG \\
\hline lacA_extd & GTCGCATGCCTATCCGGAAG \\
\hline lockP & GCCTACCGTTCGTATAGCATACATTATACGAACGGTATCCCGCTATTGCGGGAAGCGCTGCA \\
\hline Pkcol & GCGCTTCCCGCAATAGCGGGATACCGTTCGTATAATGTATGCTATACGAACGGTAGGCTGCA \\
\hline
\end{tabular}

Multiple Modifications of the B. subtilis Genome and Cre-Mediated Activation of tet $\mathrm{R}^{\text {lox72/1 }}$

B. subtilis WH558 (fig. 2a) was chosen as the initial host strain [Bertram et al., 2005]. It carries wt-tetR, driven by a constitutive promoter, integrated in the lacA locus. As a reporter gene, lac $Z$ downstream of a $\mathrm{P}_{\mathrm{xyl} / \text { tet }}$ promoter [Geissendörfer and Hillen, 1990] with two tet operators is located within amyE. First, an aphAIII gene in the WH558 genome had to be removed. Reinstating this strain's Km sensitivity was mandatory for later selection of the tlklt cassette. In anticipation of the future development of a Cre/lox system for Bacillus, the aphAIII resistance cassette used to select for WH558 had been flanked by lox66 and lox71 in an earlier study [Bertram et al., 2005]. Upon treatment with plasmid pCrePA, generated for expression of Cre recombinase in $B$. anthracis [Pomerantsev et al., 2006], a Km-sensitive descendant of WH558, designated RAB100, was obtained. The observed size reduction of the region downstream of tet $R$ in the chromosome corresponded to the length of the floxed marker gene, strongly indicating Cre mediated eviction of aphAIII. RAB100 was transformed with pWH1411BD-TLKLTextd. The integration of the $t l k l t$ fragment into tetR of RAB100 yielded strain RAB101, which was confirmed by PCR. In order to assemble and activate $t e t R^{l o x 72 / 1}$, competent RAB101 cells were treated with pCrePA (see above). PCR analyses of Km-sensitive candidates corroborated the absence of the floxed resistance cassette. One of the positive candidates, the tet $R$ region of which was confirmed by sequencing, was termed RAB102. In accordance with the expectations, Cre treatment had exactly assembled the two halves of the tet $R$-like sequence to tet $R^{\text {lox } 72 / 1}$, leaving one lox 72 scar within the $\alpha 8$ to $\alpha 9$ loop codons behind (see above). The described strain manipulation procedure is schematically summarized in figure $2 \mathrm{~b}$, with the corresponding PCR analyses shown in figure $2 \mathrm{c}$. The regulatory capacities of the newly constructed $B$. subtilis WH558 derivatives were assayed by $\beta$-gal measurements, the results of which are shown in the upper part of figure 3. No differences in $\beta$-gal activity were observed between WH558 and RAB100, which differ in the aphAIII selection marker downstream of tetR only. RAB101 shows no lacZ repression, which is explained by a stop-codon immediately downstream of lox71 in tlklt (not shown). As in the case of tet $R^{l o x 72 / 2-i n v}$, this construct thus does not form a functional repressor. For RAB102 $\left(\operatorname{tet}^{l o x 72 / 1}\right)$, an almost identical activity profile to that of WH558 or RAB100 was observed. This is in accordance with the results in E. coli (fig. 1b). To assay the amounts of TetR (or the shorter tlklt-encoded product) of the respective Bacillus strains, Western Blot analyses were conducted. Figure 3 (lower part) depicts the results obtained with the monoclonal antibody TOP19, whose epitope maps to the helix-turn- 
Fig. 1. a Structure of TetR(D). The two monomers are colored in blue and grey with the $\alpha$-helices illustrated as barrels or as the backbone trace, respectively. Tc is depicted in yellow. In the blue-colored monomer, the termini and the helices $\alpha 8$ and $\alpha 9$ are marked. The connecting loop, which is modeled in this representation, is highlighted in red. The region between the two arrows was targeted in the described approach. b Amino acid sequence alignment of different $\alpha 8-\alpha 9$ loop regions. The amino acid sequences from the five C-terminal residues of helix $\alpha 8$ to the end of helix $\alpha 9$ of TetR variants or TetR-like polypeptides are shown. Identical positions are shaded black or grey. The C-terminal end of TetR $\mathrm{R}^{\text {lox72/2-inv }}$ is followed by a diamond. The positions of two PstI sites used for cloning within the respective tet $R$ alleles are indicated by arrows. $\beta$-gal activities of the respective constructs as assayed in $E$. coli (in \%) are given on the right.
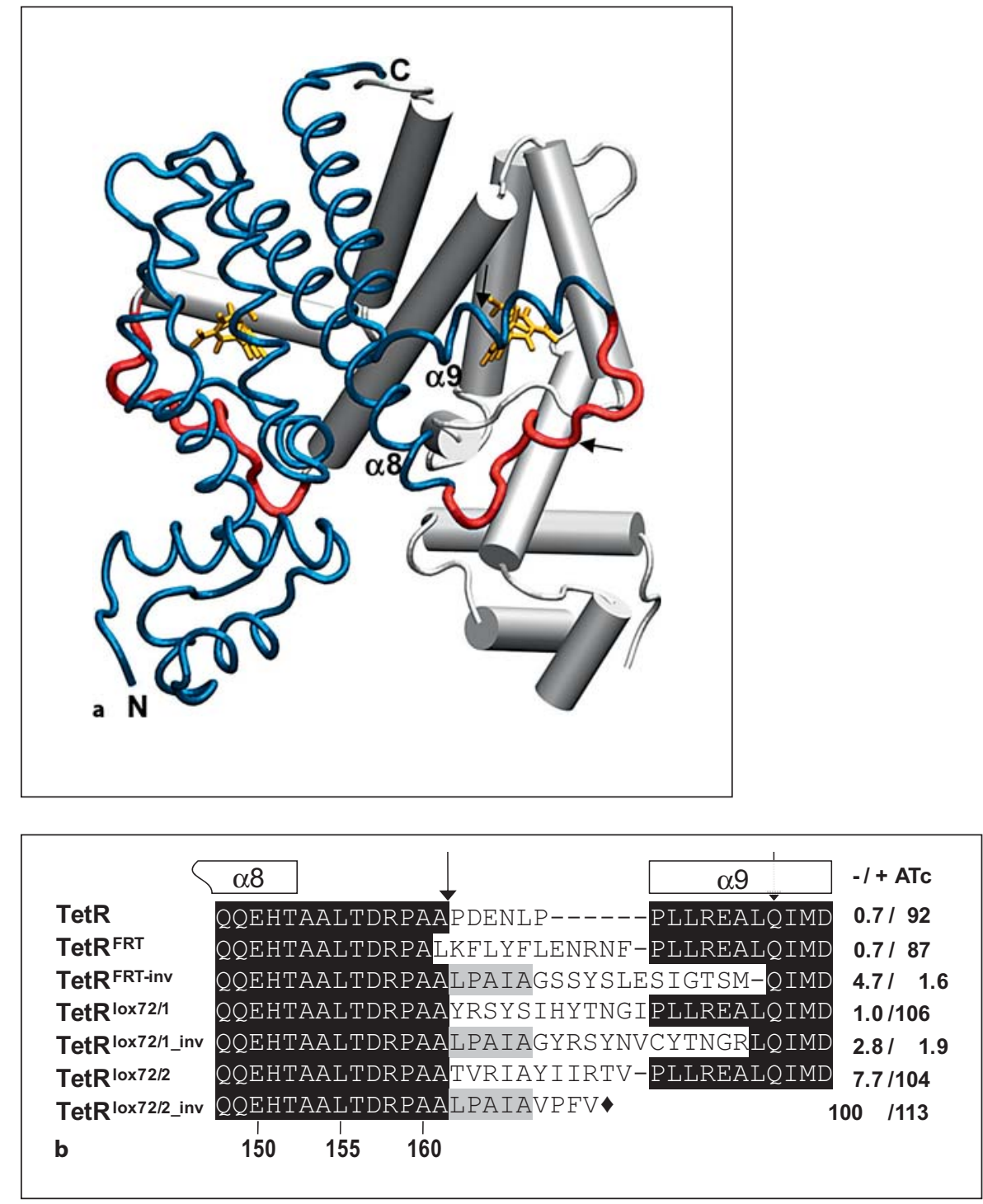

helix motif of TetR(B) [Pook et al., 1998], which is identical to that of TetR. The approximately equally strong signals obtained with soluble protein extracts of WH557, WH558 and RAB100 contrast to the weaker band in case of RAB102. No signal was observed with RAB101, which encodes the prematurely terminated TLKLT product corresponding to residues 1-161 of TetR with an additional eleven amino acids at the $\mathrm{C}$-terminus. Additional Western analyses using polyclonal antibodies raised against TetR revealed an even stronger reduction of signal intensity in RAB102, when compared to WH558 and RAB100 (not shown). This may be explained by fewer antibody recognition epitopes in the altered loop region of TetR $\mathrm{R}^{\mathrm{lox} 72 / 1}$ compared to wt-TetR. Nevertheless, the re- duced regulator amounts of RAB102 are sufficient to achieve high-capacity tet regulation in the B. subtilis model system.

\section{Discussion}

Exerting transcriptional control is the most prevalent technique for artificial gene regulation in bacteria to date. In most cases, transcription initiation is modulated by a regulator protein which conditionally binds to its cognate DNA sequence, dependent on the interaction with a suitable small ligand. For TetR we here describe an additional stage of control of higher hierarchy. The described kind 
Fig. 2. a Schematic representation of the initial B. subtilis measurement strain WH558. The locations of tetR and the $\mathrm{P}_{\mathrm{xyl} / \mathrm{tet}}$-lac $Z$ fusion are schematically depicted. Open bent arrows denote the promoters Pt17 (for tet $R$ expression) and $\mathrm{P}_{\mathrm{xyl} / \mathrm{tet}}$ (tet-regulatable, upstream of lacZ). Black trapezoids depict various lox sites as denoted, flanking the kanamycin resistance cassette (aphAIII), also indicating their direction. Boxes marked 'O' represent tet operators. A transcriptional terminator is drawn as a hairpin. Three successive stop codons are symbolized by a capital ' $\mathrm{S}$ '. The figure is not drawn to scale. b Multiple alterations of the tetR region. As a first step, the aphAIII gene was excised by Cre recombinase, leading to the marker-free strain RAB100. Secondly, the tet $R$ gene was replaced by double homologous recombination for a tet $R^{\prime}$-lox66aphAIII-lox71-tetR (tlklt) sequence, resulting in B. subtilis RAB101. Third, the aphAIII gene was once again eliminated by Cre, giving rise to the final construct RAB102 harboring tet $R^{l o x 72 / 1}$. Primers for analytic PCR reactions (see fig. 2c), indicated by small arrows and latin numbering, are: (I) lox_test_fw, (II) DP3, (III) DP8neu, (IV) Km1, (V) Km2, (VI) lox_ test_rev. The figure is not drawn to scale. c Results of analytic PCRs PCR products of the four indicated B. subtilis strains obtained with the following primer combinations (see fig. $2 \mathrm{~b}$ for approximate primer locations): lane 1: I + VI, lane 2: III + VI, lane 3: I + II and lane 4: IV + V (from left to right). Sizes of selected reference bands (in bp) are indicated.

\section{WH558}
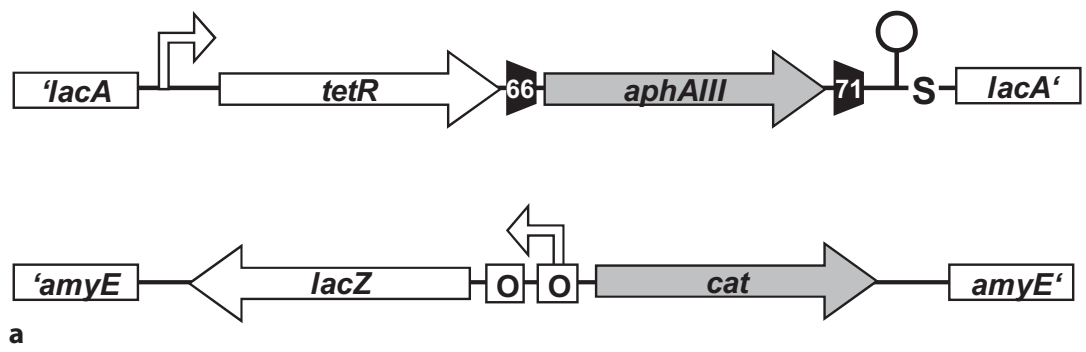

a
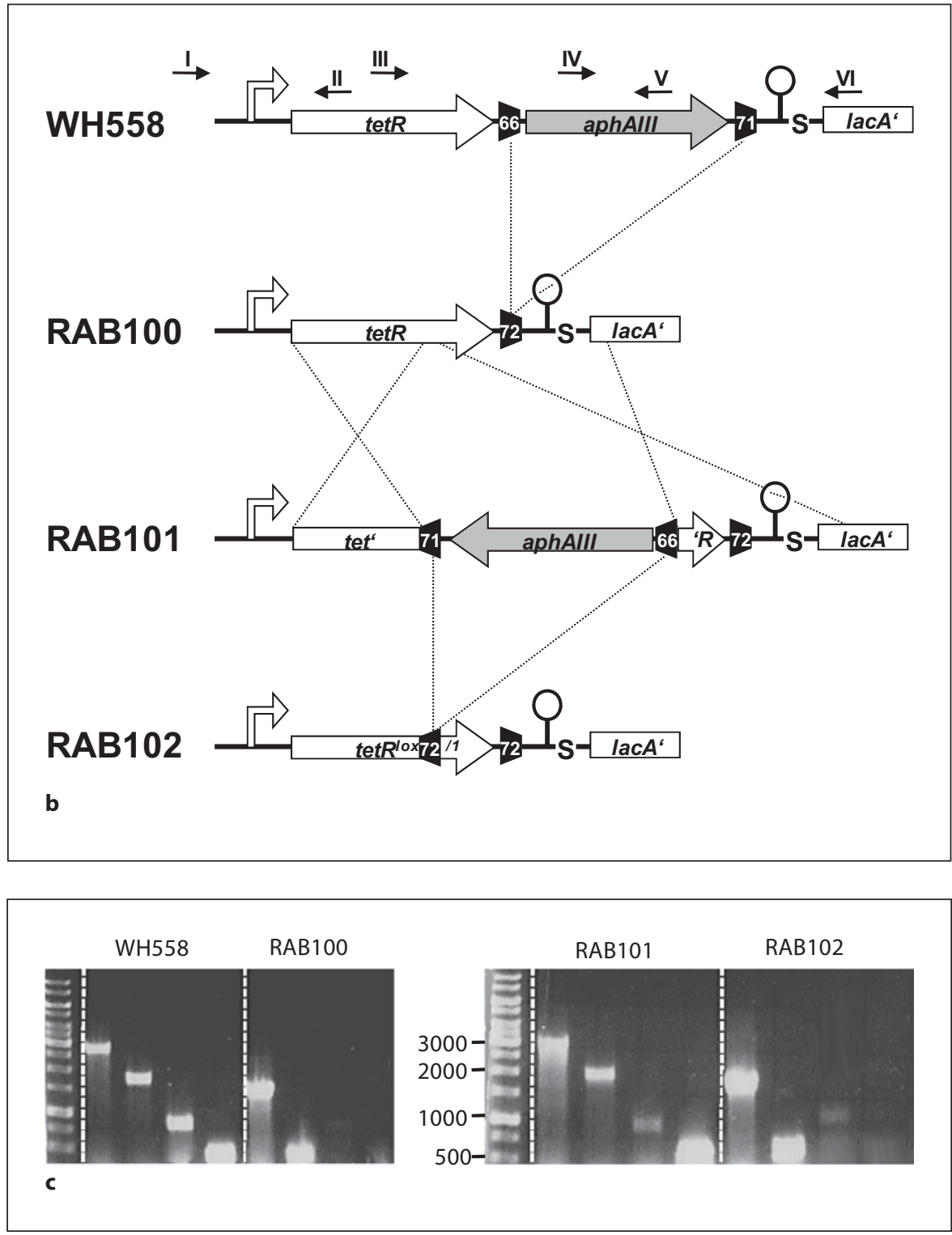
of activation abrogated undesired leaky repression of the target gene (in this study tetR), which is occasionally encountered in transcriptional regulation systems. Instead, activation of TetR was achieved at the genomic level by exploiting site-specific recombination (SSR), in this case by Cre/lox technology. A plasmid for Cre expression in $B$. anthracis [Pomerantsev et al., 2006] turned out to be very effective also in $B$. subtilis and was used to consecutively eliminate a lox66-aphAIII-lox71 cassette twice out of two adjacent genomic loci (fig. 2b). The presence of a lox72 scar after the first round of Cre treatment did not impede the second recombination between lox66 and lox71, corroborating previous findings that lox72 is a poor substrate for Cre [Albert et al., 1995; Lambert et al., 2007]. In the past, SSR has already been exploited for gene activation in bacteria. Genes in E. coli were re-oriented using Flp/FRT, so that they became attached to a promoter and thus expressed [Sektas et al., 1999; Sektas and Szybalski, 1998]. Unlike our approach and earlier studies, this method did not incorporate SSR binding sites into an ORF. The group of Boccard fused the 5'-part of lac $Z$ to $a t t L$ and the 3 '-part to $a t t R$ of phage $\lambda$. A lacZ allele harboring att $B$ could thus reversibly be assembled by the recombinases Int and Xis in E. coli. [Esnault et al., 2007; Valens et al., $2004]$. The encoded $\beta$-gal variant served the purpose of a readout for spatial proximity of distant chromosomal sites, but showed only about $50 \%$ of the wild type enzyme's activity. Kaczmarczyk and Green [2001] also described SSR-mediated $\beta$-gal activation by excision of a floxed egfp gene in order to monitor Cre activity. In the resulting protein the lox encoded residues were within a fused N-terminus, which is not part of the native enzyme. By contrast, our approach implies that a target protein must tolerate primary structure alteration. In the case of TetR, the $\alpha 8-\alpha 9$ loop seemed the most promising region to this end. Among the 15 different naturally occurring TetR orthologues known to date [Agersø and Guardabassi, 2005; Berens and Hillen, 2004; Brown et al., 2008; Thompson et al., 2007], little primary sequence conservation is observed within the loop, which is 12 to 21 amino acids long. This also holds true for the unique loops of the TetR variants described here. Scholz et al. [2001] have constructed a 'loop-less' TetR(D) variant and have also substituted this region for stretches of two to 42 mostly alanine residues. It proved to be the case that the loop per se, but not its sequence is critical for TetR(D) induction by ATc. Variants with loop lengths of about 17-29 were most efficient in that study and the 21-residue loop of TetR $R^{\text {lox } 72 / 1}$ thus fits into this range. The fact that an $[E D] R$ motif (corresponding to positions 156 and 157 of TetR),

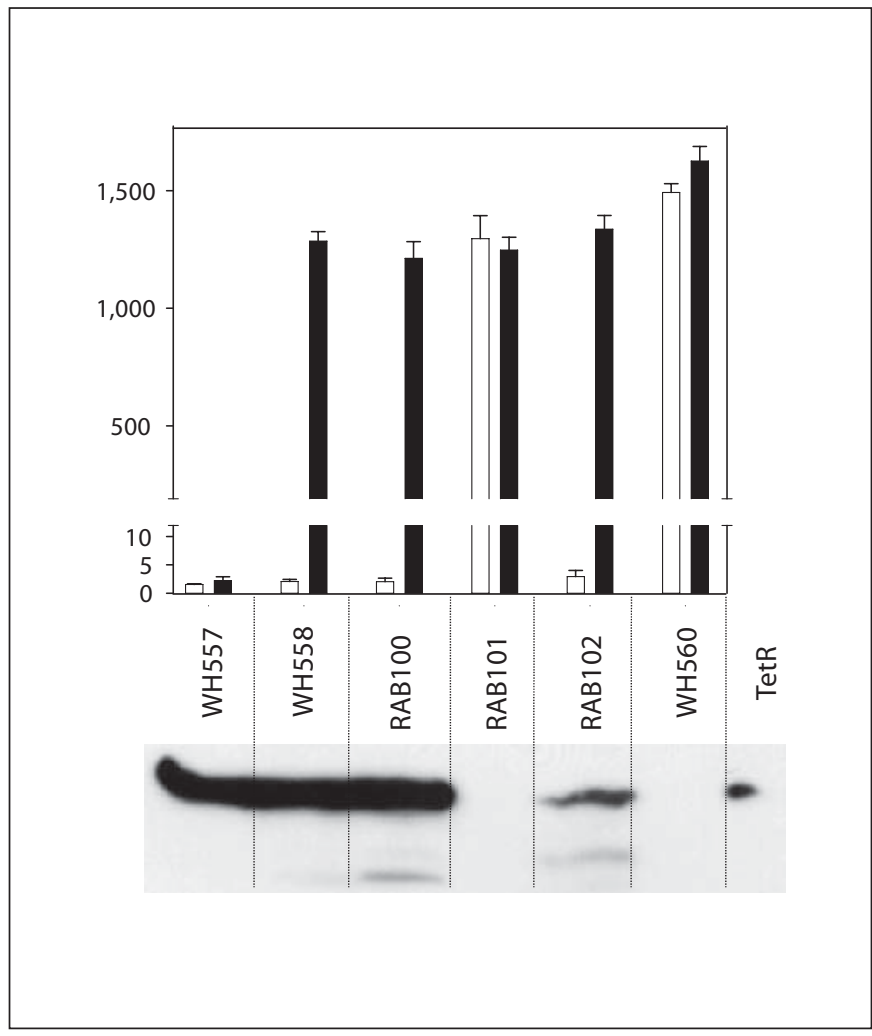

Fig. 3. Regulatory capacities and regulator amounts of different $B$. subtilis strains. Results of $\beta$-gal measurements are depicted in the upper panel, with Miller units given on the y-axis. Control strains are WH557, encoding tetR but not lacZ and WH560, which harbors $\mathrm{P}_{\mathrm{xyl} / \mathrm{tet}}$-lacZ but no tetR. Open bars depict values obtained without ATc, whereas closed bars represent $0.4 \mu \mathrm{M}$ ATc conditions. Western blot results for the corresponding strains, obtained with monoclonal antibody, are shown below. $80 \mathrm{ng}$ of purified TetR was used as a control.

which is conserved in most of the original TetR variants' loops, was maintained may have improved inducibility by ATc [Scholz et al., 2001]. Poly-alanine TetR(D) variants furnished with loops of more than 20 residues had previously shown decreased intracellular protein levels. This is in accordance with our observations of $\mathrm{Tet}^{\operatorname{lox} 72 / 1}$ (fig. 2c), which, nonetheless, displayed tight repression. The weaker in vivo repression capacities of Tet $\mathrm{R}^{\operatorname{lox} 72 / 2}$ may be rationalized by instability. The induction defects of the full-length '-inv' constructs underline the participation of helix $\alpha 9$ in forming TetR's inducer binding pocket. Interestingly, however, these constructs are stable enough to mediate repression. It can therefore be inferred that formation of the four-helix bundle of the helices $\alpha 8$ and $\alpha 10$ of both monomers, which build a rigid scaffold 
of TetR [Hinrichs et al., 1994; Kisker et al., 1995] is not impaired. Taken together, the variants TetR $\mathrm{R}^{\operatorname{lox} 72 / 1}$ and $\mathrm{TetR}^{\mathrm{FRT}}$, and also TetR $\mathrm{R}^{\text {lox72/2 }}$ to a lesser extent (fig. 1b) retained the functions of both DNA-binding and ATc inducibility. Thus, to our knowledge, the present study for the first time reports Cre/lox-mediated gene assembly resulting in the activation of a fully efficient allosteric protein, which is only slightly altered in a carefully selected permissive region. Further manipulations of tet $R$ within cells are also conceivable through the described approach. These include reversible Cre-mediated alteration or (in)activation of TetR function by inserting or excising stop codons or transcription factor encoding DNA into tet $R$ in vivo. This may enable both the generation of novel regulators with altered functions and switching TetR activity in an inducer-independent manner. Furthermore, the described mode of TetR activation can be viewed as an attractive read-out tool for monitoring the activity of SSR systems. Based upon our observations that TetR $R^{\text {lox }}$ and TetR $\mathrm{R}^{\mathrm{FRT}}$ variants displayed full efficiency, it can be assumed that TetR also tolerates further similarsized SSR recognition sites within its ORF. Thus, TetR's sequence flexibility might prove useful for analyzing activities of both the known and new SSR systems, which are as yet untapped for molecular genetics purposes.

\section{Experimental Procedures}

\section{Bacterial Strains and Growth Conditions}

Cloning was carried out in Escherichia coli DH5 $\alpha$ [Hanahan, 1983]. Genetic manipulations of tet $R$ in vivo were conducted in $B$. subtilis WH558 [Bertram et al., 2005] and derivatives thereof, as outlined in table 1 . Cells were generally grown either in liquid (shaking) or on solid LB or BM [Bera et al., 2005]. When appropriate, media where supplemented with ampicillin (Ap; $100 \mathrm{mg} / \mathrm{l}$ for E. coli), kanamycin (Km; $30 \mathrm{mg} / \mathrm{l}$ for E. coli, or $15 \mathrm{mg} / \mathrm{l}$ for B. subtilis), chloramphenicol (Cm; $25 \mathrm{mg} / \mathrm{l}$ for E. coli, or 5 or $10 \mathrm{mg} / \mathrm{l}$ for B. subtilis) or erythromycin (Em; $2.5 \mathrm{mg} / \mathrm{l}$ for B. subtilis). E. coli strains for cloning were made competent and transformed using standard techniques [Hanahan, 1983]. Naturally competent $B$. subtilis cells were obtained using an established protocol [Kraus et al., 1994]. All strains used in this study are summarized in table 1 .

\section{DNA Isolation and Modification}

Plasmid DNA was prepared from E. coli according to the manufacturers' protocols using the E.Z.N.A. Plasmid Miniprep Kit (Peqlab, Erlangen, Germany), NucleoSpin Plasmid (MachereyNagel, Düren, Germany), or the Plasmid Midi Kit (Qiagen, Hilden, Germany). For amplification of B. subtilis chromosomal DNA, colonies from solid medium were directly inoculated into a PCR reaction mix using illustra PuReTaq Ready-To-Go PCR beads (GE Healthcare, Munich, Germany). Sequencing of plasmids or PCR products was accomplished using an ABI PRISM 310 Genetic Analyzer (Applied Biosystems, Weiterstadt, Germany) or carried out at GATC (Constance, Germany). Primers were purchased from Biomers (Ulm, Germany) or MWG-Biotech (Ebersberg, Germany), longer oligonucleotides were obtained from TIB-MOLBIOL (Berlin, Germany). The sequences of the primers used for cloning or sequencing in this study are listed in table 2.

\section{Construction of Plasmids for Expression of tetR Alleles}

Carrying SSR Sites

Throughout this study, the TetR(BD) chimera which comprises the TetR(B) sequence from position 1 to 50 and residues 51 to 208 of TetR(D) was used [Schnappinger et al., 1998; Schubert et al., 2001]. For convenience, TetR(BD) is hereafter referred to as TetR. Complementary oligonucleotides containing FRT or lox sites for in-frame insertion into tet $R$ were $5^{\prime}$ phosphorylated by $\mathrm{T} 4$ polynucleotide kinase (New England Biolabs, Frankfurt/Main, Germany). Subsequently, two complementary oligonucleotides were hybridized by mixing $37.5 \mathrm{pmol}$ of each in aqueous solution, heating to $90^{\circ} \mathrm{C}$ and cooling down to RT within approximately $1 \mathrm{~h}$. The hybridized oligonucleotides carried 3' overhangs, compatible or identical to those of PstI cut DNA and contained the following SSR recognition sites: FRT within flp_fw/flp_rev, and lox72 in two different reading frames within lockP/Pkcol and lockP_fw/lockP_rev. Upon insertion into tetR of Pst I cut pWH1926 [Kamionka et al., 2006], the following desired constructs were obtained: $\mathrm{pWH} 1926-\mathrm{F}$ with FRT in its $5^{\prime} \rightarrow 3^{\prime}$ reading frame 2, pWH1926-L1 (lox72 in $3^{\prime} \rightarrow 5^{\prime}$ frame 1) and pWH1926-L2 lox72 $\left(3^{\prime} \rightarrow 5^{\prime}\right.$ frame 2$)$. In all constructs the SSR recognition sites are embedded in a tetR ORF. Plasmids in which the fragments were inserted in opposite orientation are designated correspondingly and are suffixed '-inv'.

\section{Cloning of a B. subtilis Integration Vector to Allow for}

Cre-Mediated tetR Activation

For disruption of tet $R$ with a floxed kanamycin resistance marker, an aphAIII cassette was amplified from plasmid pDG792 [Guérout-Fleury et al., 1995] by PCR using primers Km_66_inv and Km_71_inv. The PCR product was inserted into pWH1926 via PstI. Of the two possible resulting plasmids, that with aphAIII in opposite orientation to the disrupted tet $R$ was used and designated pWH1926-TLKLT (for tetR'-lox66-aphAIII-lox71-'tetR). The target strain B. subtilis WH558 carries DNA of pUC19 chromosomally, which is the ancestor of pWH1926 derivatives. To circumvent possible undesired ectopic insertion upon transformation with pWH1926-TLKLT, the tetR'-lox66-aphAIII-lox71-'tetR sequence was subcloned into pWH1411BD [Scholz et al., 2003] via BglII and NcoI, yielding pWH1411-TLKLT. To provide a larger region of sequence identity for genomic integration, a part of the chromosomal sequence within and downstream of tet $R$ was amplified from B. subtilis RAB100 DNA (table 1) using primers DP8neu and lacA_extd (table 2). This fragment was inserted into pWH1411-TLKLT via NcoI and PaeI to obtain the integration vector pWH1411-TLKLTextd, which was linearized by NheI prior to transformation of $B$. subtilis.

\section{Applied Techniques for Genome Modifications of}

B. subtilis Strains

Elimination of floxed aphAIII cassettes from B. subtilis chromosomes was achieved by treatment of the respective cells with 
pCrePA, a plasmid which was constructed for Cre expression in $B$. anthracis [Pomerantsev et al., 2006]. In general, $1 \mu \mathrm{g}$ of this plasmid was used to transform $B$. subtilis. Transformands were first cultivated at $30^{\circ} \mathrm{C}$ on media containing $2.5 \mu \mathrm{g} / \mathrm{ml} \mathrm{Em} \mathrm{but}$ lacking $\mathrm{Km}$, as the resistance marker aphAIII was intended to be eliminated. On average, at least $10 \%$ of the analyzed clones displayed $\mathrm{Km}$ resistance after pCrePA treatment. Em-resistant colonies were re-streaked on plates without Em and incubated at $37^{\circ} \mathrm{C}$, a temperature which is not permissive for pCrePA. Candidate clones were streaked out again and incubated overnight at $37^{\circ} \mathrm{C}$ a second time. Clones which displayed both Em and $\mathrm{Km}$ sensitivity after this treatment, indicating marker eviction and pCrePA loss, were checked by PCR analyses.

\section{$\beta$-Galactosidase Activity Quantifications}

In vivo repression and induction capacities of Tet $R$ variants were determined by $\beta$-galactosidase ( $\beta$-gal) assays of mid-log cultures of E. coli WH207 $\lambda$ tet50 [Smith and Bertrand, 1988; Wissmann et al., 1991] or various B. subtilis strains as described [Kamionka et al., 2005; Scholz et al., 2004]. Cells were grown in LB alone (repressive conditions), or supplemented with $0.4 \mu \mathrm{m}$ ATc (for induction of TetR). Three independent cultures were assayed, with the measurements conducted at least twice. Values obtained for $E$. coli cells carrying the tetR-less plasmid
pWH1925 [Bertram et al., 2004], mimicking full induction, were set to $100 \%$. Standard deviations of these measurements were below $10 \%$.

\section{Western Blot Analyses}

Immunodetection of TetR in soluble $B$. subtilis extracts was performed using $75 \mu \mathrm{g}$ of total protein, and either serum of TOP19 monoclonal antibody raised against TetR(B) [Pook et al., 1998] or a 1:20,000 dilution of rabbit polyclonal antibodies recognizing TetR in conjunction with ECL Plus Western Blotting Detection Reagents (GE Healthcare, Munich, Germany) as described in detail previously [Kamionka et al., 2005].

\section{Acknowledgements}

We are indebted to Stephen Leppla and Andrei Pomerantsev for kindly providing larger amounts of pCrePA. We thank Christian Berens for helpful comments and Evelyn Tichy and Rosmarie Gaupp are acknowledged for critically reading the manuscript. This work was supported by grants BE 4038 and SFB 473 of the Deutsche Forschungsgemeinschaft and the Fonds der Chemischen Industrie.

\section{References}

-Agersø Y, Guardabassi L: Identification of tet 39, a novel class of tetracycline resistance determinant in Acinetobacter spp. of environmental and clinical origin. J Antimicrob Chemother 2005;55:566-569.

Albert H, Dale EC, Lee E, Ow DW: Site-specific integration of DNA into wild-type and mutant lox sites placed in the plant genome. Plant J 1995;7:649-659.

-Bera A, Herbert S, Jakob A, Vollmer W, Götz F: Why are pathogenic staphylococci so lysozyme resistant? The peptidoglycan $o$-acetyltransferase oata is the major determinant for lysozyme resistance of Staphylococcus aureus. Mol Microbiol 2005;55:778-787.

Berens C, Hillen W: Gene regulation by tetracyclines: constraints of resistance regulation in bacteria shape tetr for application in eukaryotes. Eur J Biochem 2003;270:3109-3121.

Berens C, Hillen W: Gene regulation by tetracyclines. Genet Eng NY 2004;26:255-277.

Berens C, Schnappinger D, Hillen W: The role of the variable region in tet repressor for inducibility by tetracycline. J Biol Chem 1997;272: 6936-6942.

Bertram R, Hillen W: The application of tet repressor in prokaryotic gene regulation and expression. Microb Biotechnol 2008;1:2-16.

-Bertram R, Köstner M, Müller J, Vazquez Ramos J, Hillen W: Integrative elements for Bacillus subtilis yielding tetracycline-dependent growth phenotypes. Nucleic Acids Res 2005; 33:e153.
Bertram R, Kraft C, Wisshak S, Mueller J, Scholz $\mathrm{O}$, Hillen W: Phenotypes of combined tet repressor mutants for effector and operator recognition and allostery. J Mol Microbio Biotechnol 2004;8:104-110.

Brown MG, Mitchell EH, Balkwill DL: Tet 42, a novel tetracycline resistance determinant isolated from deep terrestrial subsurface bacteria. Antimicrob Agents Chemother 2008;52:4518-4521.

Degenkolb J, Takahashi M, Ellestad GA, Hillen W: Structural requirements of tetracyclinetet repressor interaction: determination of equilibrium binding constants for tetracycline analogs with the tet repressor. Antimicrob Agents Chemother 1991;35:1591-1595.

Esnault E, Valens M, Espéli O, Boccard F: Chromosome structuring limits genome plasticity in Escherichia coli. PLoS Genet 2007;3: e226.

Fernandez S, Ayora S, Alonso JC: Bacillus subtilis homologous recombination: genes and products. Res Microbiol 2000;151:481-486.

Geissendörfer M, Hillen W: Regulated expression of heterologous genes in Bacillus subtilis using the tn 10 encoded tet regulatory elements. Appl Microbiol Biotechnol 1990;33: 657-663.

Grindley ND, Whiteson KL, Rice PA: Mechanisms of site-specific recombination. Annu Rev Biochem 2006;75:567-605.

Grkovic S, Brown MH, Skurray RA: Regulation of bacterial drug export systems. Microbiol Mol Biol Rev 2002;66:671-701.
Guérout-Fleury AM, Shazand K, Frandsen N, Stragier P: Antibiotic-resistance cassettes for Bacillus subtilis. Gene 1995;167:335-336.

Hanahan D: Studies on transformation of Escherichia coli with plasmids. J Mol Biol 1983; 166:557-580.

Hillen W, Berens C: Mechanisms underlying expression of tn 10 encoded tetracycline resistance. Annu Rev Microbiol 1994;48:345369.

Hillen W, Schollmeier K: Nucleotide sequence of the $\operatorname{tn} 10$ encoded tetracycline resistance gene. Nucleic Acids Res 1983;11:525-539.

Hinrichs W, Kisker C, Düvel M, Müller A, Tovar K, Hillen W, Saenger W: Structure of the tet repressor-tetracycline complex and regulation of antibiotic resistance. Science 1994; 264:418-420.

Kaczmarczyk SJ, Green JE: A single vector containing modified cre recombinase and lox recombination sequences for inducible tissuespecific amplification of gene expression. Nucleic Acids Res 2001;29:E56-56.

Kamionka A, Bertram R, Hillen W: Tetracycline-dependent conditional gene knockout in Bacillus subtilis. Appl Environ Microbiol 2005;71:728-733.

Kamionka A, Majewski M, Roth K, Bertram R, Kraft C, Hillen W: Induction of single chain tetracycline repressor requires the binding of two inducers. Nucleic Acids Res 2006;34: 3834-3841. 
Kintrup M, Schubert P, Kunz M, Chabbert M, Alberti P, Bombarda E, Schneider S, Hillen $\mathrm{W}$ : Trp scanning analysis of tet repressor reveals conformational changes associated with operator and anhydrotetracycline binding. Eur J Biochem 2000;267:821-829.

-Kisker C, Hinrichs W, Tovar K, Hillen W, Saenger W: The complex formed between tet repressor and tetracycline- $\mathrm{Mg}^{2+}$ reveals mechanism of antibiotic resistance. J Mol Biol 1995;247:260-280.

Kraus A, Hueck C, Gärtner D, Hillen W: Catabolite repression of the Bacillus subtilis $x y l$ operon involves a cis element functional in the context of an unrelated sequence, and glucose exerts additional xylr-dependent repression. J Bacteriol 1994;176:1738-1745.

Lambert JM, Bongers RS, Kleerebezem M: Crelox-based system for multiple gene deletions and selectable-marker removal in Lactobacillus plantarum. Appl Environ Microbiol 2007;73:1126-1135.

Lutz R, Bujard H: Independent and tight regulation of transcriptional units in Escherichia coli via the lacr/o, the tetr/o and arac/i1-i2 regulatory elements. Nucleic Acids Res 1997; 25:1203-1210.

- Orth P, Cordes F, Schnappinger D, Hillen W, Saenger W, Hinrichs W: Conformational changes of the tet repressor induced by tetracycline trapping. J Mol Biol 1998;279:439447.

- Orth P, Schnappinger D, Hillen W, Saenger W, Hinrichs W: Structural basis of gene regulation by the tetracycline inducible tet repressor-operator system. Nat Struct Biol 2000;7: 215-219.

Pomerantsev AP, Sitaraman R, Galloway CR, Kivovich V, Leppla SH: Genome engineering in Bacillus anthracis using cre recombinase. Infect Immun 2006;74:682-693.
Pook E, Grimm S, Bonin A, Winkler T, Hillen W: Affinities of mabs to tet repressor complexed with operator or tetracycline suggest conformational changes associated with induction. Eur J Biochem 1998;258:915-922.

Sauer B: Chromosome manipulation by cre-lox recombination; in Craig NL, Craigie R, Gellert M, Lambowitz AM (eds): Mobile DNA II. Washington, ASM Press, 2002, pp 38-58.

-Schnappinger D, Schubert P, Pfleiderer K, Hillen W: Determinants of protein-protein recognition by four helix bundles: changing the dimerization specificity of tet repressor. Embo J 1998;17:535-543.

Scholz O, Henssler EM, Bail J, Schubert P, Bogdanska-Urbaniak J, Sopp S, Reich M, Wisshak S, Köstner M, Bertram R, Hillen W: Activity reversal of tet repressor caused by single amino acid exchanges. Mol Microbiol 2004;53:777-789.

Scholz O, Kintrup M, Reich M, Hillen W: Mechanism of tet repressor induction by tetracyclines: length compensates for sequence in the S8-89 loop. J Mol Biol 2001;310:979986.

Scholz O, Köstner M, Reich M, Gastiger S, Hillen $\mathrm{W}$ : Teaching tetr to recognize a new inducer. J Mol Biol 2003;329:217-227.

Schubert P, Schnappinger D, Pfleiderer K, Hillen $\mathrm{W}$ : Identification of a stability determinant on the edge of the tet repressor four-helix bundle dimerization motif. Biochemistry 2001;40:3257-3263.

Schweizer HP: Applications of the saccharomyces cerevisiae flp-frt system in bacterial genetics. J Mol Microbiol Biotechnol 2003;5: 67-77.

Sektas M, Gregorowicz M, Szybalski W: Transient conversion to reca+ phenotype to permit p1 transduction in any Escherichia coli reca- strains. Biotechniques 1999;27:911914.
Sektas M, Szybalski W: Tightly controlled twostage expression vectors employing the flp/ frt-mediated inversion of cloned genes. Mol Biotechnol 1998;9:17-24.

-Skerra A: Use of the tetracycline promoter for the tightly regulated production of a murine antibody fragment in Escherichia coli. Gene 1994;151:131-135.

Smith LD, Bertrand KP: Mutations in the tn 10 tet repressor that interfere with induction: location of the tetracycline-binding domain. J Mol Biol 1988;203:949-959.

- Thompson SA, Maani EV, Lindell AH, King CJ, McArthur JV: Novel tetracycline resistance determinant isolated from an environmental strain of Serratia marcescens. Appl Environ Microbiol 2007;73:2199-2206.

-Unger B, Klock G, Hillen W: Nucleotide sequence of the repressor gene of the ral tetracycline resistance determinant: structural and functional comparison with three related tet repressor genes. Nucleic Acids Res 1984;12:7693-7703.

-Valens M, Penaud S, Rossignol M, Cornet F, Boccard F: Macrodomain organization of the Escherichia coli chromosome. EMBO J 2004; 23:4330-4341.

Wissmann A, Wray LV Jr, Somaggio U, Baumeister R, Geissendörfer M, Hillen W: Selection for $\operatorname{tn} 10$ tet repressor binding to tet operator in Escherichia coli: isolation of temperaturesensitive mutants and combinatorial mutagenesis in the DNA binding motif. Genetics 1991;128:225-232.

Yan X, Yu HJ, Hong Q, Li SP: Cre/lox system and PCR-based genome engineering in Bacillus subtilis. Appl Environ Microbiol 2008;74: 5556-5562. 